\title{
GENERIC BIFURCATION OF REVERSIBLE VECTOR FIELDS ON A 2-DIMENSIONAL MANIFOLD
}

\author{
Marco Antonio Teixeira
}

\begin{abstract}
In this paper we deal with reversible vector fields on a 2-dimensional manifold having a codimension one submanifold as its symmetry axis. We classify generically the one parameter families of such vector fields. As a matter of fact, aspects of structural stability and codimension one bifurcation are analysed.
\end{abstract}

\section{Introduction}

Let $M$ be a $C^{\infty}$ compact orientable two-dimensional manifold and $f: M \rightarrow \mathbb{R}$ be a $C^{\infty}$ function having 0 as regular value. Call $S=$ $\left\{f^{-1}(0)\right\}, M^{+}=f^{-1}[0, \infty), M^{-}=f^{-1}(-\infty, 0]$.

Let $\varphi: M \rightarrow M$ be a $C^{\infty}$ diffeomorphism (an involution) from $M$ onto $M$, such that $\varphi \circ \varphi=\operatorname{Id}(\varphi$ is an involution) and $\operatorname{Fix} \varphi=S$. if

We say that a vector field $X$ on $M$ is $\varphi$-reversible (or simply reversible)

$$
\varphi * X=-X \circ \varphi
$$

Let $\Phi^{r}$ be the space of the $C^{r} \varphi$-reversible vector fields on $M$ endowed with the $C^{r}$-topology $(r>2)$.

The main result of this paper says that:

The set $\Sigma_{0}$ of all $C^{r} \varphi$-reversible vector fields in $M$ which are structurally stable is open and dense in $\Phi^{r}$. This set is characterized in Section 3 (see Definition 3). 
Call $\Phi_{1}=\Phi^{r}-\Sigma_{0}$ the bifurcation set of $\Phi^{r}$. There exists a $C^{r-1}$ immersed codimension-one submanifold $\Sigma_{1}$ of $\Phi^{r}$ such that:

(i) $\Sigma_{1}$ is dense in $\Phi_{1}$ (both with the relative topology);

(ii) for any $X$ in $\Sigma_{1}$, there exists a neighborhood $B_{1}$ in the intrinsic topology of $\Sigma_{1}$ such that any $Y$ in $B_{1}$ is topologically equivalent to $X$;

(iii) the part of $\Sigma_{1}$ imbedded in $\Phi^{r}$ is also characterized.

In [11], we have classified all the symmetric singularities of codimension 0,1 and 2 of $X \in \Phi^{r}$. We have presented a technique which enabled us to classify in a simple manner those singularities. In that work the treatment is local and the technique consists to make a special change of coordinates around the point and then address the analysis to the study of the contact between a general system and $S$. In this paper we follow those ideas and use extensively the tools of the Singularity Theory and the results contained in $[\mathbf{1}],[\mathbf{7}],[\mathbf{9}],[\mathbf{1 0}],[\mathbf{1 1}]$ and $[\mathbf{1 2}]$. In our setting, the strategy is to establish a connection between a reversible system on $M$ and a vector defined on $M^{+}$. Roughly speaking, having reduced the system to the study of vector fields defined in manifolds with boundary, the next step is to employ the results and methods contained in [10].

Denote by $\chi^{r}$ the space of all $C^{r}$ vector fields on $M$, endowed with the $C^{r}$ topology.

In the class of all vector fields in $M$, the structural stability has been characterized by Peixoto [9]. Sotomayor [7] has characterized the structural stability of one-parameter families of vector fields in $M$. Devaney [3] has stated a Kupka-Smale Theorem for reversible vector fields and flows.

In the class of reversible vector fields some persistent phenomena occur which cannot be destroyed by perturbations in $\Phi^{r}$ such as periodic orbits and saddle connections which meet the submanifold $S$. However, concerning non trivial recurrences no surprises arise at all. As a matter of fact, this point becomes in some sense simpler in this class. We mention for example that such reversible systems on the torus do not admit an irrational flow.

We suggest that the reader see [4] for further references and connections with other problems.

This paper is organized as follows.

In Section 2 we give definitions and recall standard facts. 
Section 3 is devoted to the study of the structural stability in $\Phi^{r}$. Some considerations concerning structural stability in manifolds with boundary are made.

In Section 4 the main result of the paper is presented. In our procedure we classify the stable one parameter families of reversible vector fields on $M$.

Section 5 contains an appendix where the main tools required for the proof of the main theorem are recalled.

\section{Preliminaries}

In what follows we list some known properties of a vector field $X \in \Phi^{r}$ :

- The phase portrait of $X$ is symmetric with respect to $S$. So the knowledge of the phase portrait of $X$ in $M^{+}$determines the phase portrait of $X$ in $M$.

- If $p \in S$ and $X(p) \neq 0$ then the orbit of $X$ is always transverse to $S$ at $p$.

- If $X(p)=0$ then $X(\varphi(p))=0$. If $p \in S$ then it is called a symmetric critical point of $X$. Otherwise it is an asymmetric critical point. Moreover $\varphi$ interchanges the stable and unstable manifolds and a symmetric critical point cannot be an attractor or a repellor.

- Any periodic orbit of $X$ crossing $S$ is called a symmetric periodic orbit of $X$. Any symmetric periodic orbit is never an isolated limit cycle. If, on the other hand, a periodic orbit $\gamma$ of $X$ is away from $S$ it is called asymmetric and it is paired by another periodic solution given by $\varphi(\gamma)$.

- If $X_{t}$ denotes the flow associated to $X$ then we have the following expressions:

$X_{t} \circ \varphi=\varphi \circ X_{-t}, U_{t}^{2}=\operatorname{Id}$ and $\operatorname{Fix}\left\{U_{t}\right\}=X_{t / 2}(\operatorname{Fix}\{\varphi\})$, where $U_{t}=X_{t} \circ \varphi$.

- Any orbit of $X$ connecting two asymmetric hyperbolic saddles (and so meeting $S$, transversally) is persistent under perturbation of $X$ in $\Phi^{r}$.

- There is no isolated periodic orbit of $X$ passing through points $p \in S$.

- The codimension zero (generic) symmetric critical points are either a hyperbolic saddle (with real eigenvalues $\lambda$ and $-\lambda$ ) or of 
elliptical type (with eigenvalues $\pm i b$ ). Their normal forms (see for example [11]) are:

$$
X_{01}(x, y)=(y, x) \quad \text { and } \quad X_{02}(x, y)=(-y, x) .
$$

We may refer to them as generic $S$-singularities.

- In $[2]$ it is shown the following expressions relating the genus $g$ of $M$ and the Betti number $k$ of $S$ :

(i) $k \leq g+1$,

(ii) $k \neq g \operatorname{Mod}(2)$ and

(iii) $g-k+1 \in 2 \mathbb{N}$.

In particular, one deduces that $k=1$ or 2 provided that $M$ is the sphere or the torus respectively. When $M$ is the bitorus then $k$ can be 1 or 2 .

We denote by $T=T(S)$ a neighborhood of $S$ in $M$, such that $\varphi(T)=T$ and $T^{ \pm}=T \cap M^{ \pm}$.

\section{Structural Stability}

\subsection{Structural stability in manifolds with boundary.}

Let $\chi^{r}$ be the space of the $C^{r}$ vector fields on $M$ endowed with the $C^{r}$-topology $(r>2)$.

The results in this section will be used in the sequel.

Let $Z \in \chi^{r}$ and $N$ be a 2-dimensional submanifold of $M$ with $S=\partial N$.

We say that $p \in S$ is an $S$-singularity of $Z \in \chi^{r}$ if either $Z(p)=0$ or $Z(p) \neq 0$ and $Z f(p)=0$.

It should be mention that $Z f(p)=D f(p)(Z(p))$ is still a real function defined on $M$. So we may define inductively the function $Z^{k} f(p)=$ $Z\left(Z^{k-1} f(p)\right)$ which expresses the order contact between the vector field $Z$ and the curve $S$ at $p$.

Definition 1. We say that $p \in S$ is a fold singularity of $Z$ if $Z(p) \neq 0$, $\mathrm{Z} f(p)=0$ and $Z Z f(p) \neq 0$. In this case we say that the contact between the orbit of $Z$ and $S$ at $p$ is quadratic.

A vector field $Z$ in $\chi^{r}$ is $S$-stable if there are neighborhoods $B$ of $Z$ in $\chi^{r}$ and $V$ of $N$ in $M$ such that for every $Y$ in $B$ there is an $S$-preserving homeomorphism $h(Y): V \rightarrow V$ which is a $C^{0}$ equivalence between $\left.Z\right|_{V}$ and $\left.Y\right|_{V}$. Such a homeomorphism will be refered to as an $S$-equivalence between $Z$ and $Y$. The concept of $S$-structural stability in $\chi^{r}$ is given in a natural way. 
Definition 2. Let $\Xi_{0}(S)$ be the class of all $Z$ in $\chi^{r}$ for which the following conditions are satisfied:

(0) $Z$ does not have nontrivial recurrent trajectories;

$\left(I_{1}\right)$ all critical points of $\left.Z\right|_{N}$ are hyperbolic;

$\left(I_{2}\right)$ all periodic orbits of $\left.Z\right|_{N}$ are hyperbolic;

$\left.\left(I_{3}\right) Z\right|_{N}$ does not have saddle connections;

$\left(S_{1}\right)$ all singular points of $\left.Z\right|_{N}$ are contained in the interior of $N$;

$\left(S_{2}\right)$ all periodic orbits of $\left.Z\right|_{N}$ are contained in the interior of $N$;

$\left(S_{3}\right)$ any tangency between a trajectory of $\left.Z\right|_{N}$ and $S$ is quadratic;

$\left.\left(S_{4}\right) Z\right|_{N}$ does not have tangency connections;

$\left.\left(S_{5}\right) Z\right|_{N}$ does not admit a connection between a saddle critical point and a tangency point.

Theorem 3.1 (Andronov-Pontryaguin and Peixoto). (a) $\Xi_{0}(S)$ is open and dense in $\chi^{r}$; (b) $Z$ is $S$-structurally stable in $\chi^{r}$ if only if it belongs to $\Xi_{0}(S)$.

Assume that $Z \in \Xi_{0}(S)$. A separatrix of $Z$ is an orbit which connects either two saddle critical points or two tangency points between the vector field and $S$ or a tangency point and a saddle critical point. Any equivalence between two vector fields in $\chi^{r}$ must preserve such objects.

Consider $Z \in \chi^{r}$, and $p \in N$. The positive (resp. negative) limit set of an orbit $\gamma(p)$ of $\left.Z\right|_{N}$ is the set $L^{+}(p)$ (resp. $\left.L^{-}(p)\right)$ of points $q \in N$ which are limit points of sequences of the form $\phi_{X}\left(p, t_{n}\right)$ with $t_{n}$ tending to $\omega(p)(\operatorname{resp} . \alpha(p))$.

\subsection{Structural stability of reversible vector fields.}

Let $X \in \phi^{r}$. The coming construction will be useful in the sequel.

\subsubsection{A construction.}

On a small tubular neighborhood $T_{i}$ of each connected component $S_{i}$ of $S$ in $M$, consider the following coordinates $\left(\theta_{i}, \rho_{i}\right)$ with $S_{i}=\left\{\rho_{i}=0\right\}$ and $0 \leq \theta_{i} \leq 1$ and $\left|\rho_{i}\right|<\varepsilon$.

Let $\varphi_{i}$ be the restriction of $\varphi$ on $T_{i}$. In the above coordinates,

$$
\varphi_{i}\left(\theta_{i}, \rho_{i}\right)=\left(\alpha_{i}\left(\theta_{i}, \rho_{i}\right), \beta_{i}\left(\theta_{i}, \rho_{i}\right)\right)
$$

satisfies:

$$
\rho_{i} \beta_{i}\left(\theta_{i}, \rho_{i}\right)<0 \text { and } \varphi_{i}\left(\theta_{i}, 0\right)=\left(\theta_{i}, 0\right)
$$


Define now the germ of a $C^{\infty}$ mapping at $S_{i}$,

$$
F_{i}: T_{i}, S_{i} \rightarrow \Re^{2}
$$

by

$$
F_{i}\left(\theta_{i}, \rho_{i}\right)=\left(\theta_{i}+\alpha_{i}\left(\theta_{i}, \rho_{i}\right), \theta_{i} \alpha_{i}\left(\theta_{i}, \rho_{i}\right)+\rho_{i} \beta_{i}\left(\theta_{i}, \rho_{i}\right)\right) .
$$

Proposition 3.2. Each $F_{i}$ is a fold mapping at $\left(\theta_{i}, 0\right)$ and $F_{i} \circ \varphi_{i}=F_{i}$.

Proof: Neglecting the subscripts we have:

$$
D \varphi(\theta, 0)=\left(\begin{array}{cc}
1 & b \\
0 & -1
\end{array}\right)
$$

where $b=\alpha_{\rho}(\theta, 0)$.

By a straightforward calculation we get

$$
D F(\theta, 0)=\left(\begin{array}{cc}
2 & b \\
2 \theta & b \theta
\end{array}\right) \text {. }
$$

The function $\triangle(\theta, \rho)=\operatorname{Det}(D F(\theta, \rho))$ satisfies:

$$
\triangle(\theta, 0)=0 \quad \text { and } \quad \triangle_{\theta}(\theta, 0)=-4-b^{2}<0 .
$$

So the curve $K=\operatorname{Kern}(D F(\theta, 0))=\{(u, v) ; 2 u+b v=0\}$ is transverse to the curve $\Gamma(F)=\{(u, v) ; \triangle(u, v)=0\}$.

It is immediatee to get $F \circ \varphi=F$.

This finishes the proof.

On each $T_{i}$ consider the coordinates $(\theta, \rho)$ and $\varphi=(\alpha, \beta)$ given above. On the half "plane " $\rho>0$, let

$$
u=\theta+\alpha \quad \text { and } \quad v=\theta \alpha+\rho \beta
$$

In the new coordinates, $S$ is expressed by $v=\left(\frac{u}{2}\right)^{2}$, and $X$ is transformed in $X^{*}(u, v)$ in such a way that $X^{*}\left(u,\left(\frac{u}{2}\right)^{2}\right)=0$. We finally define the vector field $H=H(X)$ on the region $F\left(T_{i}\right)$ by

$$
H(u, v)=\frac{X^{*}}{v-\left(\frac{u}{2}\right)^{2}} .
$$




\subsubsection{Local settings.}

i) Let $p \in S$ and $X \in \Phi^{r}$.

It is well known (Montgomery-Bochner Theorem in [6]) that the involution $\varphi$ at $p$, is $C^{\infty}$ conjugated to $\varphi(x, y)=(x,-y)$ at $p_{0}=0$. In this section we carry out the analysis on $\Re^{2}, 0$ and fix $f(x, y)=y$.

Observe that in local coordinates around a point $p \in S$ we have the following expressions:

$$
S=\{(x, y) ; y=0\} \quad \text { and } \quad \varphi(x, y)=(x,-y) .
$$

We may choose $F(x, y)=\left(x, y^{2}\right)$ and from the reversibility properties of the vector field, it takes the form

$$
X(x, y)=\left(y a\left(x, y^{2}\right), \frac{b\left(x, y^{2}\right)}{2}\right) .
$$

Moreover by $u=x$ and $v=y^{2}$ we derive that:

$$
H(u, v)=(a(u, v), b(u, v)) \text { for } v>0 .
$$

ii) In these coordinates the trajectory of $X$ passing through a regular point is always "orthogonal" to $S$. At a tangency point (resp. critical point), the contact between the orbit through $p$ (resp. an invariant manifold) and $S$ decays by a factor of $\frac{1}{2}$ in comparison with the orbit or invariant manifold of $H(X)$ passing through the same point. Following these considerations, we denote by $H(s)$ a trajectory of $H(X)$ corresponding to a trajectory $s$ of $X$.

Remark 3.3. Observe that $H$ can be $C^{\infty}$ extended to a full neighborhood of $p$. Moreover, due to the symmetry properties of $X$, we deduce that the behavior of $H(X)$ near $S$ determines completely the behavior of $X$ in a small neighborhood $T$ of $S$. It follows that in $\rho>0, X$ is topologically equivalent to $H(X)$. This leads us to analyse the $S$-stability of $H$ on the region $H(T)$ with boundary $S$. Moreover, outside a small neighborhood $V$ of $S$ in $T^{+}, X$ and $H(X)$ are $C^{r}$ conjugated.

Remark 3.4. It should be mentioned that if $p \in S$ is a fold point of $H(X)$ then it is a codimension 0 (generic) critical point of $X$. It is of saddle type (resp. elliptical type) provided that it is a internal (resp. external) tangency. 


\subsubsection{The manifolf $\Sigma_{0}$.}

Denote by $X^{+}$the restriction of $X$ to $C l\left\{M^{+}\right\}$.

Definition 3. We say that $X \in \Phi^{r}$ is simple if the following conditions are satisfied:

(0) $X$ does not have nontrivial recurrent trajectories;

(i) all asymmetric critical points of $X$ are hyperbolic;

(ii) all asymmetric periodic orbits of $X$ are hyperbolic;

(iii) $X^{+}$does not have saddle connections.

(iv) all symmetric singularities of $X$ are of codimension 0 .

Remark 3.5. As pointed out, if $p$ is a saddle critical point of $X$ we have to distinguish in our analysis the cases where $p$ is in $S$ or not. In the first case, it corresponds to a generic (interior) contact between $H(X)$ and $S$, and the eigenvalues associated to $D X(p)$ are $\pm \lambda$.

Remark 3.6. If $X$ is simple we may find, from [10], a tubular neighborhood $T(S)$ of $S$ in $M$ such that $X^{*}=\left.X\right|_{M^{+}-T(S)}$ satisfies the following conditions:

(0) $X^{*}$ does not have nontrivial recurrent trajectories;

(i) all critical points and periodic orbit of $X^{*}$ are hyperbolic and contained in the interior of $M^{+}-T(S)$;

(ii) $X^{*}$ does not have saddle connections;

(iii) if $X^{*}(p)$ is tangent to $\partial T(S)$ then this contact is quadratic.

In other words, we mean that $X^{*}$ satisfies the conditions of structural stability of vector fields defined on manifolds with boundary given in [8]. This auxiliar vector field is very useful in the proof of the main results of this paper.

The following result is an immediate consequence of Theorem 3.1 and Proposition 3.2.

Theorem 3.7. $X \in \Sigma_{0}$ if and only if $X$ is simple. Moreover, $\Sigma_{0}$ is open and dense in $\Phi^{r}$. 
Proof: First of all, we observe that the above conditions (0), (i), (ii), (iii) coincide exactly with the characterization of the Morse-Smale Vector Fields on $M$. It should be mentioned that those saddle separatrices, asymmetric orbits and asymmetric critical points appear in pairs (each one in a different connected component of $M-S$ ). The symmetric singularities of $X$ are studied by the auxiliar vector field $H(X)$ (see Proposition 3.2). As a matter of fact they correspond to quadratic tangencies between $H(X)$ and $S$.

Due to the symmetry properties of $X$, it is clear that all the analysis can be performed via $X^{+}$. The results and techniques, contained in [8] and [10], involving the generic contact between $H(X)$ and $S$ must be used here. We observe that the question involving recurrences is answered exactly in the same way as in the usual theory (see [5]). So it is straightforward, from Theorem 3.1 and Proposition 3.2 to get that $X$ is structurally stable provided that it is a simple vector field. So the genericity of $\Sigma_{0}$ in $\Phi^{r}$ becomes evident.

Assume for instance that $X$ violates some condition given in Definition 3. Once again we appeal to Theorem 3.1 and to Proposition 3.2 and conclude that $X$ cannot be structurally stable. In fact:

a) If $X$ has non-hyperbolic critical points or periodic orbits outside $S$ then we use standart techniques to approximate it by $Y$ in $\Phi^{r}$ having just hyperbolic critical points or periodic orbits outside $S$. We proceed similarly when we have a saddle connection off $S$.

b) Assume now that $X$ has a non codimension zero critical point $p \in S$. This implies that $H(X)$ and $S$ have a degenerated contact at $p$. Again, we perturb $X$ by getting $Y$, such that $H(Y)$ has in a neighborhood of $p$ in $M^{+}$just generic contact and/or hyperbolic critical points outside $S$ (see $[\mathbf{1 0}]$ and $[\mathbf{1 1}]$ ).

This finishes the proof.

\section{Bifurcation set}

\subsection{Generic bifurcation in manifolds with boundary.}

Let $\chi_{1}$ be the complement of $\Xi_{0}(s)$ in $\chi^{r}$. Assume that $Z \in \chi_{1}$.

Definition 4. We say that $p \in S$ is a cusp singularity of $Z$ if $Z(p) \neq 0$, $Z f(p)=0$. 
Definition 5. An $S$-hyperbolic critical point of $Z$ is a critical point $p$ in $S$ such that: (i) it is a hyperbolic critical point of $Z$; (ii) the eigenvalues of $D Z(p)$ are pairwise distinct and the corresponding eigenspaces are transversal to $S$ at $p$ and (iii) each pair of non complex conjugate eigenvalues of $D Z(p)$ have distinct real part.

A codimension one $S$-singularity of $\left.Z\right|_{N}$ is either a cusp singularity or an $S$-hyperbolic critical point $p$ in $S$. In the second case we distinguish the cases where $p$ is a node, a saddle or a focus.

In $[\mathbf{1 1}]$ it is proved the following result:

Theorem 4.1. For $r>3$, there is a codimension one $C^{r-1}$ immersed submanifold $\Xi_{1}(S)$ in $\chi^{r}$, dense in $\chi_{1}$, in such a way that for any $Z \in \Xi_{1}$, there exists a neighborhood $B$ in the intrinsic topology of $\chi_{1}$ such that for any $Y$ in $B,\left.Y\right|_{N}$ is topologically equivalent to $\left.Z\right|_{N}$. Furthermore, the part of $\Xi_{1}$ imbedded in $\chi^{r}$ is also characterized.

The characterizations of the submanifolds given in the last theorem are given in the appendix (Section 5).

Remark 4.2. Given $Z \in \chi_{1}$ the following orbits have to be distinguished: a) an invariant manifold of a saddle critical point $p \in S$; b) a strong invariant manifold of a nodal critical point $p \in S$; c) an orbit of $Z$ tangent to $S$ at $p$. Any $C^{0}$ equivalence between two elements of $\chi^{r}$ must necessarily preserve such objects. We may refer to them as $S$-separatrices of $Z$.

\subsection{Generic bifurcation of reversible vector fields.}

\subsubsection{Codimension one symmetric critical points.}

Let $X$ be in $\Phi^{r}$ and $p \in S$.

Definition 6. We say that $p$ is a codimension one symmetric singularity of $X$ if $p$ is a codimension one $S$-singularity of $H(X)$. 
There are essentially four different topological types of a codimension one symmetric singularity (see $[\mathbf{1 1}]$ ). They are:

Cuspidal type: $p$ is a cusp point of $H(X)$ (see Figure 1). Its normal form is: $X_{0}(x, y)=\left(y, x^{2}\right)$.

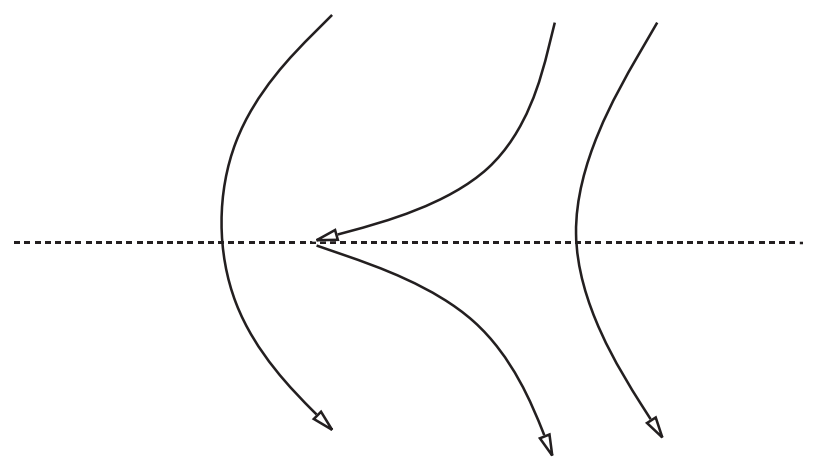

Figure 1. Cuspidal singularity.

Nodal type: $p$ is an $S$-hyperbolic critical point of $H(X)$ of nodal type (see Figure 2). Its normal form is: $X_{0}(x, y)=\left( \pm x y, x \pm 2 y^{2}\right)$.

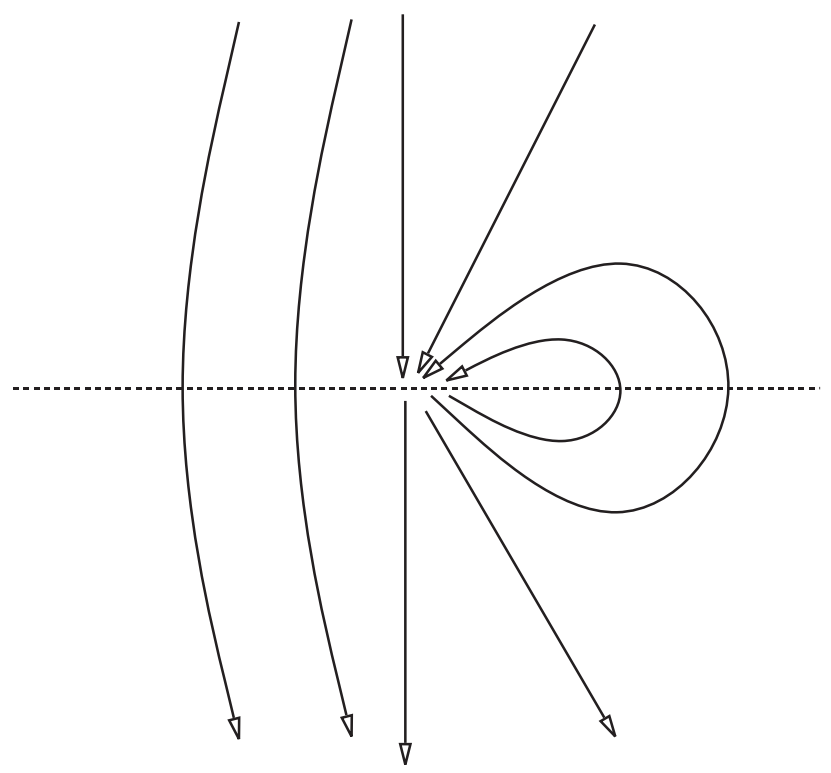

Figure 2. Nodal type singularity. 
Saddle type: $p$ is an $S$-hyperbolic critical point of $H(X)$ of saddle type (see Figure 3$)$. Its normal form is: $X_{0}(x, y)=\left(x y, x-y^{2}\right)$.

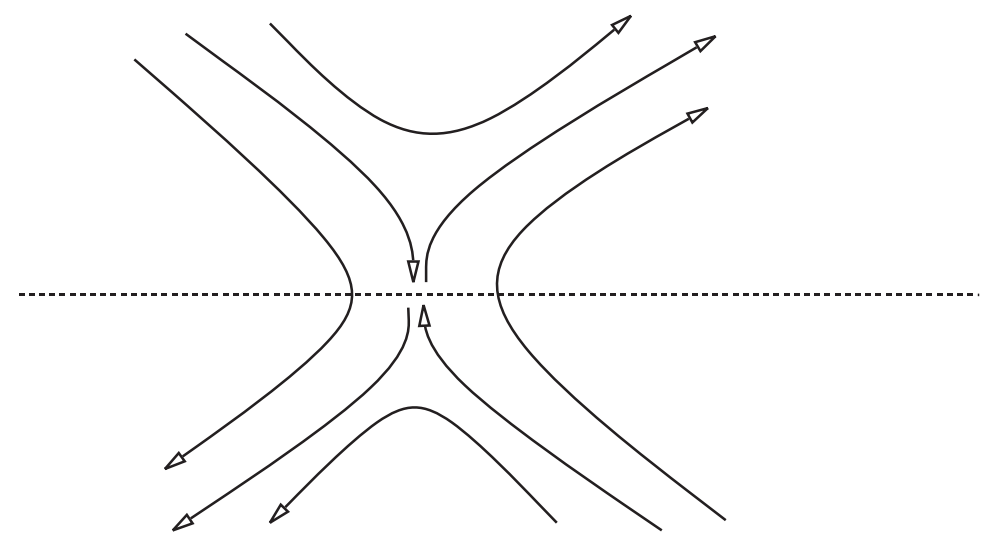

Figure 3. Saddle type singularity.

Focal type: $p$ is an $S$-hyperbolic critical point of $H(X)$ of focal type (see Figure 4). Its normal form is: $X_{0}(x, y)=\left(x y+y^{3},-x+y^{2}\right)$.

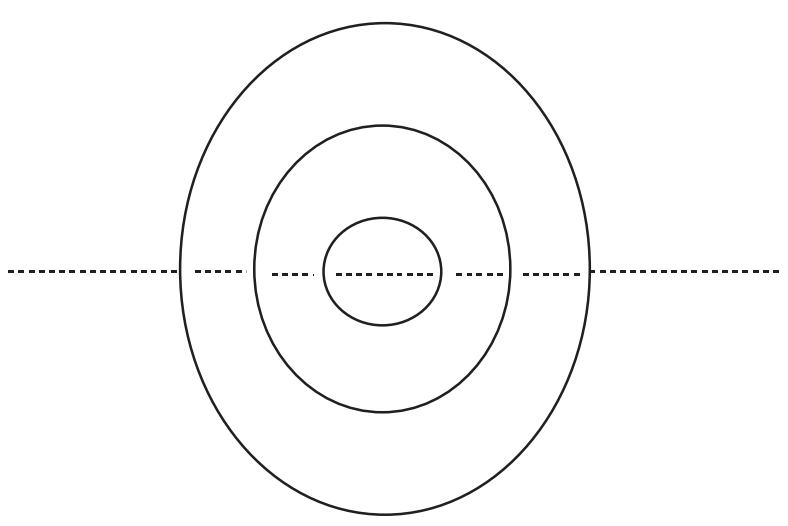

Figure 4. Focal type singularity. 
Remark 4.3. Let $p \in S$ be a codimension one symmetric singularity of $X \in \Phi^{r}$. Recalling Remark 4.2,s is a separatrix of $X$ at $p$, provided that $H(s)$ is an $S$-separatrix of $H(X)$ through $p$. It is clear that when $p$ is an $S$-critical point of $X$ of nodal type then associated to it there is a separatrix $s$ which corresponds to the strong invariant manifold of $H(X)$ at the point.

\subsubsection{Bifurcation set.}

Let $\Phi_{1}$ be the complement of $\Sigma_{0}$ in $\Phi^{r}$. Assume that $X \in \Phi_{1}$.

The set $\Phi_{1}$ is the union of the following sets of vector fields $\Phi_{1}(1)$, $\Phi_{1}(2), \Phi_{1}(3)$ and $\Phi_{1}(4)$ where conditions (i), (ii), (iii) and (iv) given in Definition 3 are violated respectively.

Call $\Sigma_{1}(1)$ the set of vector fields $X$ in $\Phi_{1}(1)$ such that $X^{+}$has a unique non-hyperbolic critical point $p_{X}$ in the interior of $\mathrm{M}^{+}$(i.e. asymmetric critical point). Moreover it is a codimension one critical point of $X$ (i.e. a saddle-node or a generic Hopf singularity) and all the other conditions (0), (ii), (iii) and (iv) in Definition 3 are satisfied.

Call $\Sigma_{1}(2)$ the set of vector fields $X$ in $\Phi_{1}(2)$ such that $X^{+}$has a unique non-hyperbolic (asymmetric) periodic orbit $\gamma(X) \subset \operatorname{Int}\left(M^{+}\right)$. Moreover it is a codimension one periodic orbit of $X$ (i.e. a semi stable periodic orbit) and all the other conditions (0), (i), (iii) and (iv) in Definition 3 are satisfied.

Denote by $\Sigma_{1}(3)$ the set of vector fields $X$ in $\Phi_{1}(3)$ such that $X^{+}$has a unique saddle connection $\nu(X)$. Moreover all the other conditions (0), (i), (ii) and (iv) in Definition 3 are satisfied.

Call $\Sigma_{1}(4)$ the set of vector fields $X$ in $\Phi_{1}(4)$ such that $X^{+}$has a unique non-generic critical point in $S$ (i.e. a symmetric critical point). Moreover: a) it is a codimension one symmetric critical point of $X$, b) all the other conditions (i), (ii) and (iii) in Definition 3 are satisfied. It should be mentioneded that condition b) implies in particular that if $p$ is of nodal type then no separatrix of $X^{+}$at $p$ converges to a saddle.

Consider the set

$$
\Sigma_{1}=\cup_{j=1, \ldots, 4} \Sigma_{1}(j) .
$$

We have still to define the following subsets.

Let $\Sigma_{1}^{0}(2)$ be the subset of $\Sigma_{1}(2)$ constituted by elements $X$ which satisfy the following extra assumptions:

(i) there exists no $q \in M^{+}-\gamma(X)$, such that $L^{+}(q)=L^{-}(q)=\gamma(X)$;

(ii) there exist no saddle points $s_{i}$ of $X^{+}$in $M^{+}, i=1,2$ such that

$$
L^{+}\left(W^{u}\left(s_{1}\right)\right)=L^{-}\left(W^{s}\left(s_{2}\right)\right)=\gamma(X) .
$$


We denote by $\Sigma_{1}^{0}(3)$ the subset of $\Sigma_{1}(3)$ constituted by the elements $X$ such that the saddle connection is a homoclinic orbit $\nu(X)$. In this case we impose that no other separatrix of $X^{+}$tends to $\nu(X)$.

Finally we define

$$
\Sigma_{1}^{0}=\Sigma_{1}(1) \cup \Sigma_{1}^{0}(2) \cup \Sigma_{1}^{0}(3) \cup \Sigma_{1}(4) .
$$

In what follows we present three lemmas which prepare the way for the proof of the main theorem of the paper. Their proofs are slight variations of the proof of the main result of Sotomayor in [7].

Lemma 4.4. $\Sigma_{1}(1)$ is a $C^{r-1}$ imbedded codimension one submanifold of $\Phi^{r}$. Moreover, every $X \in \Sigma_{1}(1)$ has a neighborhood $B$ in $\Phi_{1}$ such every $Y \in B$ is $C^{0}$ equivalent to $X$.

Proof: Given $X \in \Sigma_{1}(1)$ we define the auxiliar vector field $X^{*}$ (as in Remark 3.6) in such a way that this latter system satisfies the Proposition 2.1 of $[\mathbf{1 0}]$. Now define:

a) The set of $X \in \Phi_{1}$ such that: $X^{*}$ has a codimension one critical point as its unique non hyperbolic critical point and $X^{*}$ satisfies the conditions (0), (ii) and (iii) of Definition 3;

b) The set $\mathrm{H}_{0}$ of elements $X \in \Phi^{r}$ which are $S$-elementaries;

c) The set $\Lambda_{0}$ of elements $X \in \Phi^{r}$ such that $X^{+}$has no saddle connection.

Observe that $\mathrm{H}_{0}$ and $\Lambda_{0}$ are open sets of $\Phi^{r}$ and it can be proved (in $[7])$ that $\Gamma_{1}^{*}$ is a $C^{r-1}$ imbedded codimension one submanifold of $\Phi^{r}$. As $\Sigma_{1}(1)=\Gamma_{1}^{*} \cap H_{0} \cap \Lambda_{0}$, the first part of lemma is immediate. Moreover, since around the codimension one critical point is locally structurally stable relative to $\Phi_{1}$, using standard technique (see for example [10]) the proof of second part becomes straightforward.

Remark 4.5. It is well known how $X$ in $\Sigma_{1}(1)$ unfolds generically around the saddle-node or the Hopf singularity. In the case of a saddlenode if the saddle separatrix meets $S$ then it presents a (symmetric) saddle-node connection $s$ which is persistent under perturbation of $X$ in $\Phi_{1}$; when we unfold the saddle-node we get in a neighborhood of $s$ in $M$ either a hyperbolic saddle connection or a family of symmetric periodic orbits (this phenomenon is illustrated in Figure 5). 

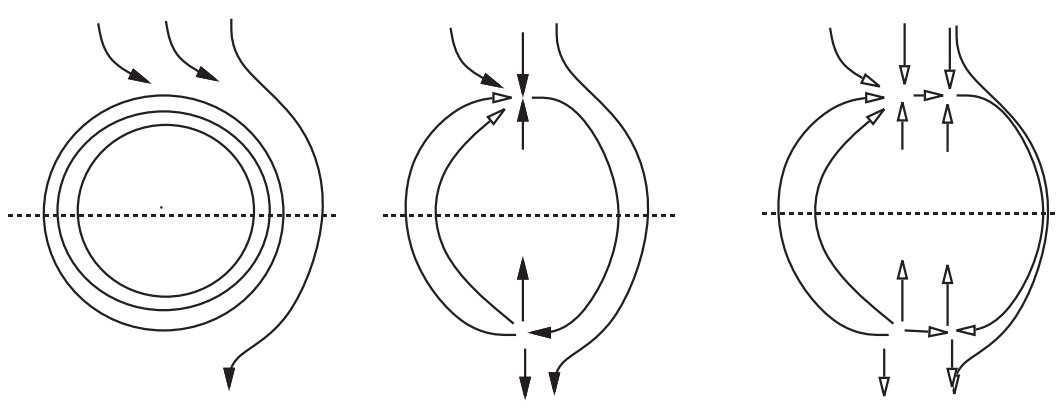

Figure 5. Symmetric sadle-node connection.

The proof of next lemma is similar to that of the last result and it will be omitted.

Lemma 4.6. i) $\Sigma_{1}(2)$ is a $C^{r-1}$ immersed codimension one submanifold of $\Phi^{r}$. Moreover, every $X \in \Sigma_{1}(2)$ has a neighborhood $B$ in $\Sigma_{1}(2)$ such that every $Y \in B$ is $C^{0}$ equivalent to $X$; ii) $\Sigma_{1}^{0}(2)$ is a $C^{r-1}$ imbedded codimension one submanifold of $\Phi^{r}$. Moreover, every $X \in \Sigma_{1}^{0}(2)$ has a neighborhood $B$ in $\Phi_{1}$ such every $Y \in B$ is $C^{0}$ equivalent to $X$.

Remark 4.7. As an illustration we mention that if $X \in \Sigma_{1}(2)-\Sigma_{1}^{0}(2)$ in such a way that there are saddle separatrices $s_{1}$ and $s_{2}$ of $X$ with $\alpha\left(s_{1}\right)=\omega\left(s_{2}\right)=\gamma_{X}$ then we may find a sequence $X_{n} \in \Sigma_{1}(3)$ converging to $X$ in $\Phi_{1}$.

Lemma 4.8. i) $\Sigma_{1}(3)$ is a $C^{r-1}$ immersed codimension one submanifold of $\Phi^{r}$. Moreover, every $X \in \Sigma_{1}(3)$ has a neighborhood $B$ in $\Sigma_{1}(3)$ such every $Y \in B$ is $C^{0}$ equivalent to $X$; ii) $\Sigma_{1}^{0}(3)$ is a $C^{r-1}$ imbedded codimension one submanifold of $\Phi^{r}$. Moreover, every $X \in \Sigma_{1}^{0}(3)$ has a neighborhood $B$ in $\Phi_{1}$ such every $Y \in B$ is $C^{0}$ equivalent to $X$.

Proof: Let $X \in \Sigma_{1}(3)$ and $\nu^{+}(X)$ be its saddle connection at $p_{1}$ and $p_{2}$ in $M^{+}$. Let $\nu^{-}(X), p_{1}$ and $p_{2}$ in $M^{+}$be similar objects in $M^{-}$and $\nu(X)=\nu^{+}(X) \cup \nu^{-}(X)$.

We consider $B$ and $V$ small neighborhoods of $X$ in $\Phi^{r}$ and of $\nu(X)$ in $M$ respectively, such that the conditions (0), (i), (ii), (iii) and (iv) of Definition 3.

We distinguish the cases:

(a) If the saddles do not belong to $S$ then the proof follows immediately from in $[\mathbf{7}]$. 
(b) Assume that $p_{1} \in S$ and $p_{2} \notin S$ in such a way that $W^{u}(p-1)=$ $W^{s}\left(p_{2}\right)=\nu^{+}(X)$. Choose neighborhoods $B_{1}, U_{1}$ and $U_{2}$ of $X$ in $\Phi^{r}, p_{1}$ in $M,\left\{p_{1}, p_{2}\right\}$ in $M$ respectively, such that $Y$ has a unique saddle point in $U_{1} \cap S$, unique saddle point in $U_{2} \cap M^{+}$. Furthermore $W^{u}(p-1)$ (resp. $W^{s}(p-2)$ ) is transverse to $\partial U_{1}$ at $m_{1} \in \operatorname{Int}\left(M^{+}\right)$(resp. $\partial U_{1}$ at $\left.n_{1} \in \operatorname{Int}\left(M^{+}\right)\right)$and transverse to $\partial U_{2}$ at $m_{2} \in \operatorname{Int}\left(M^{+}\right)\left(\operatorname{resp} . \partial U_{2}\right.$ at $\left.n_{2} \in \operatorname{Int}\left(M^{+}\right)\right)$. Hence, $Y \in \Sigma_{1}(3)$ if and only if $m_{1}=n_{1}$. Now standart techniques (see in $[\mathbf{1 0}]$ ) allows us to finish the proof of the lemma in the present case.

(c) if both points $p_{1}$ and $p_{2}$ are in $S$, the proof is very similar to the above case.

Remark 4.9. As an illustration assume for instance that $X \in \Sigma_{1}(3)-$ $\Sigma_{1}^{0}(3)$ in such a way that there is a homoclinic orbit $\nu(X)$ at $p_{X}$ and a saddle separatrix s converging to this orbit. Then we may find a sequence $\mathrm{X}_{n} \in \Sigma_{1}(3)$ converging to $X$ in $\Phi_{1}$ (see Figure 6). Observe that such $p_{X}$ has to be necessarily in $\operatorname{Int}\left(M^{+}\right)$.
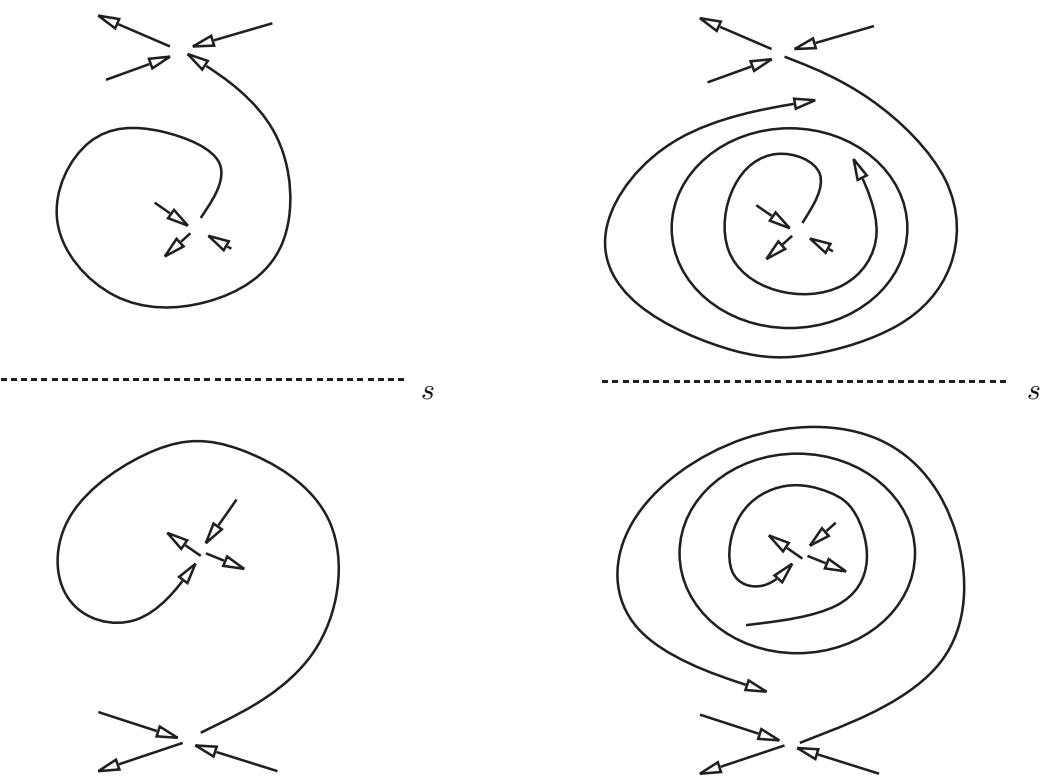

$X_{n}$

$X_{0}$

Figure 6. Asymmetric semi-stable periodic orbit. 
The following result is obtained from [11] by using the same techniques of the proof of Lemma 4.8 which have been developed in [10].

Lemma 4.10. $\Sigma_{1}(4)$ is a $C^{r-1}$ imbedded codimension one submanifold of $\Phi^{r}$. Moreover, every $X \in \Sigma_{1}(4)$ has a neighborhood $B$ in $\Phi_{1}$ such every $Y \in B$ is $C^{0}$ equivalent to $X$.

Theorem 4.11. i) $\Sigma_{1}$ is dense in $\Phi_{1}$ (both with the relative topology);

ii) for any $X$ in $\Sigma_{1}$, there exists a neighborhood $B_{1}$ in the intrinsic topology of $\Sigma_{1}$, such that any $Y$ in $B_{1}$ is topologically equivalent to $X$

iii) $\Sigma_{1}^{0}$ is the part of $\Sigma_{1}$ imbedded in $\Phi^{r}$;

iv) In the space of one parameter families of vector fields in $\Phi^{r}$, let $\Theta$ be the collection of elements $\xi(\lambda)$ (with $|\lambda|<\varepsilon$ ), such that: a) $\xi(\lambda) \subset \Sigma_{0} \cup \Sigma_{1}^{0}$; b) $\xi$ is transversal to $\Sigma_{1}^{0}$. Then any family $\xi$ is structurally stable if and only if $\xi \in \Theta$.

Proof: This proof follows from the definitions of $\Sigma_{1}$ and $\Sigma_{1}^{0}$ and from the Lemmas 4.4, 4.6, 4.8 and 4.10.

Corollary 4.12. In the space of one parameter families of vector fields in $\Phi^{r}$, let $\Theta$ be the collection of elements $\xi(\lambda)$ (with $|\lambda|<\varepsilon$ ), such that: a) $\xi(\lambda) \subset \Sigma_{0} \cup \Sigma_{1}^{0}$; b) $\xi$ is transversal to $\Sigma_{1}^{0}$. Then any family $\xi$ is structurally stable if and only if $\xi \in \Theta$.

\section{Appendix}

In this section we recall some aspects of the main result in [10] concerning the structural stability of one parameter families of vector fields defined in manifolds with boundary.

The bifurcation set $\chi_{1}$ in $\chi^{r}$ is the union of the following sets of vector fields $\chi_{1}\left(I_{1}\right), \chi_{1}\left(I_{2}\right), \chi_{1}\left(I_{3}\right), \chi_{1}\left(S_{1}\right), \chi_{1}\left(S_{2}\right), \chi_{1}\left(S_{3}\right), \chi_{1}\left(S_{4}\right)$ and $\chi_{1}\left(S_{5}\right)$ where the conditions $\left.\left.\left.\left.\left.\left.\left.I_{1}\right), I_{2}\right), I_{3}\right), S_{1}\right), S_{2}\right), S_{3}\right), S_{4}\right)$ and $\left.S_{5}\right)$ given in the definition of the set $\Xi_{0}$, are violated respectively.

Call $\Xi_{1}\left(I_{1}\right)$ the set of vector fields $X$ in $\chi_{1}\left(I_{1}\right)$ such that $\left.Z\right|_{N}$ has a unique non-hyperbolic critical point outside $S$. Moreover it is a codimension one critical point of $Z$ (i.e. a saddle-node or a generic Hopf singularity) and all the other conditions $\left.\left.\left.\left.\left.I_{2}\right), I_{3}\right), S_{1}\right), S_{2}\right), S_{3}\right), S_{4}$ ) and $\left.S_{5}\right)$ in Definition 1 are satisfied. 
Call $\Xi_{1}\left(I_{2}\right)$ the set of vector fields $Z$ in $\chi_{1}\left(I_{2}\right)$ such that $\left.Z\right|_{N}$ has a unique non-hyperbolic periodic orbit $\gamma(Z)$. Moreover it is a codimension one periodic orbit of $Z$ (i.e. a semi stable periodic orbit) and all the other conditions $\left.\left.\left.\left.\left.\left.I_{1}\right), I_{3}\right), S_{1}\right), S_{2}\right), S_{3}\right), S_{4}\right)$ and $S_{5}$ ) in Definition 1 are satisfied.

We denote by $\Xi_{1}^{0}\left(I_{2}\right)$ the subset of $\Xi_{1}\left(I_{2}\right)$ constituted by the elements $Z$ which satisfy: $i$ ) there exists no $q \in N-\gamma(Z)$, such that $L^{+}(q)=$ $L^{-}(q)=\gamma(Z)$; b) there exists no saddle points $s_{i}$ in $N, i=1,2$ such that $L^{+}\left(W^{u}\left(s_{1}\right)\right)=L^{-}\left(W^{s}\left(s_{2}\right)\right)=\gamma(Z)$; c) associated to $Z$ there exists no $(s, q) \in N \times N$, where $s$ is a saddle point of $Z, q \in S$, and $Z(q)$ is tangent to $S$ at this point, with $L^{+}(q)=L^{-}(q)=\gamma(Z)$; there exist no $p_{i} \in S$ with $L^{+}\left(p_{1}\right)=L^{-}\left(p_{2}\right)=\gamma(Z)$.

Denote by $\Xi_{1}\left(I_{3}\right)$ the set of vector fields $Z$ in $\chi_{1}\left(I_{3}\right)$ such that $\left.X\right|_{N}$ has a unique saddle connection $\nu(Z)$. Moreover all the other conditions $I_{1}$ ), $\left.\left.\left.\left.\left.I_{2}\right), S_{1}\right), S_{2}\right), S_{3}\right), S_{4}\right)$ and $S_{5}$ ) in Definition 1 are satisfied.

We denote by $\Xi_{1}^{0}\left(I_{3}\right)$ the subset of $\Xi_{1}\left(I_{3}\right)$ constituted by the elements $X$ such that the saddle connection is a homoclinic orbit. Moreover no trajectory of $\left.Z\right|_{N}$ which is either tangent to $S$ or a saddle separatrix tends to $\nu(Z)$.

Denote by $\Xi_{1}\left(S_{1}\right)$ the set of vector fields $Z$ in $\chi_{1}\left(S_{1}\right)$ such that $\left.Z\right|_{N}$ has a unique critical point $p_{Z}$ in $S$ and: a) $p_{Z}$ is $S$-hyperbolic and of nodal type, b) there is no separatrix which is the strong manifold of $p_{Z}$ and c) all the other conditions $\left.\left.\left.\left.\left.\left.I_{1}\right), I_{2}\right), I_{3}\right), S_{2}\right), S_{3}\right), S_{4}\right)$ and $S_{5}$ ) in Definition 1 are satisfied.

Denote by $\Xi_{1}\left(S_{2}\right)$ the set of vector fields $Z$ in $\chi_{1}\left(S_{2}\right)$ such that $\left.Z\right|_{N}$ has a unique periodic orbit $\gamma(Z)$ tangent to $S$, while all the other conditions $\left.\left.\left.\left.\left.\left.I_{1}\right), I_{2}\right), I_{3}\right), S_{1}\right), S_{3}\right), S_{4}\right)$ and $\left.S_{5}\right)$ in Definition 1 are satisfied.

We denote by $\Xi_{1}^{0}\left(S_{2}\right)$ the subset of $\Xi_{1}\left(S_{2}\right)$ constituted by the elements $Z$ such that $\gamma(Z)$ is neither the $\alpha$ nor the $\varpi$ limit, of either the saddle separatrices or the trajectories tangent to $S$ (with respect to $\left.Z\right|_{N}$ ).

Denote by $\Xi_{1}\left(S_{3}\right)$ the set of vector fields $Z$ in $\chi_{1}\left(S_{3}\right)$ such that $\left.Z\right|_{N}$ has a unique orbit tangent to $S$ and this tangency point is at a cusp point. Moreover all the other conditions $\left.\left.\left.\left.\left.I_{1}\right), I_{2}\right), I_{3}\right), S_{1}\right) S_{2}\right), S_{4}$ ) and $\left.S_{5}\right)$ in Definition 1 are satisfied.

Denote by $\Xi_{1}\left(S_{4}\right)$ the set of vector fields $Z$ in $\chi_{1}\left(S_{4}\right)$ such that $\left.Z\right|_{N}$ has a unique orbit tangent to $S$ in more than one point. Moreover this orbit contains exactly 2 tangency points and all the other conditions $I_{1}$ ), $\left.\left.\left.\left.I_{2}\right), I_{3}\right), S_{1}\right), S_{2}\right), S_{3}$ ) and $S_{5}$ ) in Definition 1 are satisfied. 
Denote by $\Xi_{1}\left(S_{5}\right)$ the set of vector fields $Z$ in $\chi_{1}\left(S_{5}\right)$ such that $\left.Z\right|_{N}$ has a unique saddle separatrix tangent to $S$. Moreover all the other conditions $\left.\left.\left.\left.\left.I_{1}\right), I_{2}\right), I_{3}\right), S_{1}\right), S_{2}\right), S_{3}$ ) and $S_{4}$ ) in Definition 1 are satisfied.

We define $\Xi_{1}$ as the union of the sets $\Xi_{1}\left(I_{j}\right)$ and $\Xi_{1}\left(S_{l}\right)$ for $j=1,2,3$ and $k=1,2,3,4,5$.

The part of $\Xi_{1}$ imbedded in $\chi^{r}$ is:

$\Xi_{1}^{0}=\Xi_{1}\left(I_{1}\right) \cup \Xi_{1}^{0}\left(I_{2}\right) \cup \Xi_{1}^{0}\left(I_{3}\right) \cup \Xi_{1}\left(S_{1}\right) \cup \Xi_{1}^{0}\left(S_{2}\right) \cup \Xi_{1}\left(S_{3}\right) \cup \Xi_{1}\left(S_{4}\right) \cup \Xi_{1}\left(S_{5}\right)$.

\section{References}

1. A. Andronov, E. Leontovich, I. Gordon and A. Maier, Theory of bifurcations of dynamical systems on a plane, Israel Program for Sc. Translations, Jerusalem (1971).

2. R. Cruz, Reflections on closed manifolds, Preprint IMECC/ UNICAMP (1996).

3. R. Devaney, Reversible diffeomorphisms and flows, Trans. Amer. Math. Soc. 218 (1976), 89-113.

4. J. S. W. Lamb, Reversing Symmetries in Dynamical Systems, Thesis, University of Amsterdam (1994).

5. W. De Melo and J. Palis, "Geometric Theory of Dynamical Systems, an introduction," Springer Verlag, NY, 1982.

6. D. Montgomery And L. ZipPin, "Topological Transformations Groups," Interscience, NY, 1955.

7. J. Sotomayor, Generic one parameter families of vector fields on 2-dimensional manifolds, Publ. IHES 43 (1974), 5-46.

8. M. C. Peixoto And M. M. Peixoto, Structural stability in the plane with enlarged conditions, An. Acad. Brasil. Ciênc. 31 (1959), $136-160$.

9. M. M. Pеiхото, Structural stability on two-dimensional manifolds, Topology 1 (1962), 101-120.

10. M. A. TeIXeIRA, Generic bifurcation in manifolds with boundary, J. Differential Equations 25(1) (1977), 65-89.

11. M. A. Teixeira, Singularities of reversible vector fields, Phys. $D$ 100 (1997), 101-118.

12. S. M. Vishik, Vector fields near the boundary of a manifold, Vestnik 
Moskov. Univ. Ser. Mat. Mekh. 27(1) (1972), 21-28.

1991 Mathematics subject classifications: 58F14, 34C23

\author{
IMECC-UNICAMP \\ Caixa Postal 6065 \\ 13081-970 Campinas \\ BRASIL \\ e-mail: teixeira@ime.unicamp.br
}

Primera versió rebuda el 30 de Novembre de 1996, darrera versió rebuda el 21 d'Abril de 1997 